\section{El empoderamiento de adultos mayores organizados en la búsqueda de un nuevo contrato social: experiencias del Banco Interamericano de Desarrollo y la Red Tiempos}

\author{
Tomás Engler ${ }^{1}$
}

Palabras clave: ancianos, salud del anciano, organizaciones.

\footnotetext{
Banco Interamericano de Desarrollo, División de Programas Sociales 1, 1300 New York Ave., Washington, D.C., 20577, Estados Unidos de América. Correo electrónico: tomase@iadb.org
}

Es bien conocido que la baja prioridad que se da a la vejez en las políticas nacionales obliga a los adultos mayores a depender más de su propio esfuerzo, organización y protagonismo, así como del apoyo solidario de la familia y de la comunidad, para satisfacer sus necesidades básicas.

La sociedad civil vinculada a la vejez en América Latina y el Caribe - tanto las organizaciones de adultos mayores como las dedicadas a servicios específicos para este segmento de la poblacióndesempeña un importante papel en la defensa de sus intereses y en la batalla por lograr que la sociedad y los propios adultos mayores tengan más conciencia de los problemas que enfrentan las personas de mayor edad. Además, llenan muchos vacíos que aún subsisten en la oferta de servicios públicos dirigidos a los adultos mayores. En todos los países de América Latina y el Caribe, los adultos mayores organizados han demostrado su activismo ante el Estado y sus posibilidades, capacidad y fuerza como conductores y protagonistas de su propio desarrollo y bienestar. Los aportes materiales, intelectuales y espirituales que hacen a sus familiares y a la sociedad - como jefes de familia, líderes comunitarios y trabajadores productivos- son expresiones palpables de ese potencial.

Aunque se desconoce el número exacto de agrupaciones y redes de adultos mayores en América Latina y el Caribe, solo en cinco países sudamericanos (Argentina, Chile, Colombia, Perú y Uruguay) funcionan más de 30000 organizaciones de este tipo, agrupadas en no menos de 1000 redes (1-3). Entre estas organizaciones hay clubes barriales y municipales de adultos mayores, asociaciones gremiales de jubilados, grupos artísticos, organizaciones de abogacía y cabildeo, agrupaciones políticas y organizaciones culturales y de turismo. En varios países de la Región se han establecido consejos nacionales de adultos mayores (por ejemplo, en Argentina, Brasil y Costa Rica) y se ha logrado la aprobación de leyes que refrendan derechos y beneficios específicos para las personas de mayor edad (por ejemplo, en Argentina, Bolivia, Brasil, Chile, Costa Rica y Perú). Su presencia y beligerancia - a veces con el apoyo de las defensorías del pueblo- siguen siendo fundamentales para velar por el cumplimiento de las leyes vigentes y eliminar la discriminación por razones de edad.

En los países centroamericanos también hay redes de organizaciones de adultos mayores. $\mathrm{La}$ Asociación Gerontológica Costarricense (AGECO) 
agrupa 10 redes provinciales con más de 100 organizaciones de base (Z. Esquivel, comunicación personal, 2004). Por su parte, la Asociación Nacional del Adulto Mayor de Honduras (ANAMH) cuenta con cincuenta agrupaciones afiliadas y el apoyo de la Red Nacional Hondureña de Adultos Mayores (RENHAM), una organización no gubernamental (ONG) dedicada a trabajar a favor de los adultos mayores (J. Torres y G. Gaviria, comunicación personal, 2004). En Nicaragua, la Fundación Universidad de la Tercera Edad (FUNITE) ha organizado 13 agrupaciones de adultos mayores en distintas ciudades del país y - aun cuando no todas se encuentran activas- operan junto a otras organizaciones, como la Fundación Nicaragüense para el Envejecimiento (FUNIVE), la Asociación Nicaragüense de Jubilados y la Asociación de Jubilados del Sector Salud (AJUSS), entre otras (H. López, comunicación personal, 2004). En El Salvador funcionan ocho organizaciones de base de adultos mayores y 42 entidades que ofrecen servicios a este sector de la población, aunque en la actualidad no están agrupadas en una red que coordine sus esfuerzos ( $\mathrm{S}$. Artola, comunicación personal, 2004). En la República Dominicana, la Red por una Vejez Digna agrupa alrededor de 12 organizaciones (R. Pereyra, comunicación personal, 2004).

Sin embargo, las acciones y la propia existencia de estas organizaciones y redes se ven afectadas por debilidades gerenciales y financieras y por los bajos niveles de participación observados en algunos países como Chile (14\%), Argentina (menos de 30\%), Uruguay (alrededor de 25\%) o Perú (incipiente). Además, carecen de apoyo gubernamental, lo que limita el ejercicio pleno de los deberes y derechos ciudadanos de los adultos mayores. Más importante aun, estas restricciones frenan la concertación de un nuevo contrato social intergeneracional que consagre las nuevas reglas de convivencia exigidas por el cambio demográfico que estamos viviendo.

Se puede vislumbrar un pacto solidario entre generaciones que amplíe el capital social colectivo y defina un equilibrio más justo entre los derechos y los deberes ciudadanos de todos los grupos de edad, tanto de los niños como de los jóvenes y de los adultos de mediana y mayor edad. Para los grupos organizados de adultos mayores, este pacto solidario implica ir más allá de la denuncia y de la reivindicación, y asumir un papel activo en la solución de sus propios problemas y necesidades. Es precisamente el cumplimiento de esos deberes cívicos lo que les daría mayor fuerza moral para exigir, negociar y lograr -en consenso con el Estado y con otros actores sociales- más apoyo para la población de mayor edad. Entre los compromisos que se pueden alcanzar está, por ejemplo, que los adultos mayores adopten un estilo de vida más saludable e independiente que les permita continuar haciendo aportes productivos, tanto en el ámbito laboral como social. Por su parte, el resto de la sociedad se podría comprometer a conocer mejor a los adultos mayores, a apreciarlos, tratarlos y aceptarlos como una fuerza social, económica, cultural, educativa, ética y política, y a eliminar la discriminación por razones de edad. Un pacto de estas características traería consigo una nueva cultura de vida para todos y una vejez fructífera, digna, activa, productiva y saludable para todas las personas.

Ante las debilidades descritas, es urgente y oportuno tomar medidas para vencer las limitaciones que impiden transformar estos grupos y redes en organizaciones prósperas, dinámicas y autosostenibles, capaces de asumir - junto con otros elementos de la sociedad civil y del Estado- mayores responsabilidades y protagonismo en la tarea de garantizar la vigencia de su propia ciudadanía.

\section{PROYECTO RED TIEMPOS}

En este contexto, el Banco Interamericano de Desarrollo (BID) decidió financiar un proyecto presentado por la Red Tiempos y sus filiales en Argentina, Chile, Perú y Uruguay y otras entidades públicas de esos países. Esta iniciativa, que se puso en marcha en el año 2002, tenía como propósito incrementar la afiliación, capacidad e influencia de las redes y organizaciones participantes con miras a fortalecer la demanda, la oferta y la gestión de programas que atienden a las necesidades de las organizaciones de adultos mayores y de sus miembros. Para ello se contó con una donación de US\$750 000,00 del Fondo Especial Japonés, administrado por el BID, que se sumó al equivalente de US\$ 330 000,00 aportados en especie por las organizaciones participantes. Este apoyo se aprobó en el marco de la Agenda para el Envejecimiento Exitoso, del BID, que con la consigna de "digno, activo, productivo y saludable" apoya especialmente líneas de acción dirigidas a: 1) promover una nueva cultura de la vejez y un nuevo contrato social intergeneracional; 2 ) fortalecer las redes y las organizaciones de adultos mayores, su participación e inserción social; 3) formular e implantar políticas y programas públicos y privados que apoyen el buen envejecimiento; 4) fortalecer la seguridad económica de los adultos mayores más vulnerables, especialmente el acceso a empleos, a acciones de apoyo y a créditos para iniciativas propias; 5) promover y facilitar espacios públicos y viviendas acogedoras, seguras y accesibles; y 6) ayudar a los ancianos a conservar la autonomía física y mental y promover el envejecimiento en el hogar.

El objetivo general del proyecto Red Tiempos es contribuir a reducir la pobreza y a mejorar la ca- 
lidad de vida de los adultos mayores de bajos ingresos. Para ello promueve la participación de estos en organizaciones y redes que los ayuden a satisfacer sus necesidades básicas y a mejorar su integración social y económica.

Este proyecto trabaja específicamente para establecer una red regional de organizaciones vinculadas a los adultos mayores que brinde asistencia técnica, capacitación y otros servicios dirigidos a mejorar la capacidad de las redes nacionales participantes. A escala nacional, los objetivos del proyecto son: a) fortalecer las organizaciones afiliadas en materia de gestión, oferta de servicios, control de proyectos y captación de nuevos miembros; $b$ ) coordinar con instituciones públicas el trabajo dirigido a mejorar la atención de los adultos mayores; c) recaudar fondos que garanticen la continuidad de los servicios que prestan a sus afiliados; $\mathrm{y} d$ ) promover en sus respectivos países actitudes sociales positivas hacia los adultos mayores, así como estilos de vida saludables y políticas conducentes a una vida activa, productiva y sana en los adultos mayores pobres. La Red Tiempos apoyó la elaboración de proyectos de apoyo a las acciones anteriores que pudieran recibir financiamiento del BID.

\section{La Red Tiempos y las redes nacionales}

La Red Tiempos, creada en 1996 bajo el auspicio de Caritas, está afiliada a la red mundial HelpAge Internacional. Actualmente está compuesta de 13 redes que agrupan a más de 200 organizaciones de adultos mayores o dedicadas a su servicio en 11 países (Argentina, Bolivia, Brasil, Chile, Colombia, Ecuador, Honduras, Nicaragua, Perú, República Dominicana y Uruguay). ${ }^{2}$ La Red Chilena de Programas para el Adulto Mayor proporciona la sede temporal, aunque la presidencia y las funciones de secretaría general son rotativas.

En la creación del proyecto participaron seis redes $\mathrm{u}$ organizaciones nacionales que se describen brevemente a continuación.

Argentina. La Red Mayor, creada con el apoyo de este proyecto bajo la tutela de la Fundación ISALUD, de la Argentina, reúne cerca de 60 organizaciones y redes y cuenta con un periódico (Lazos)

\footnotetext{
2 Argentina: Red Mayor; Bolivia: Mesa Defensa del Anciano y Consejo de Venerables Ancianos; Brasil: Centro Internacional de Envejecimiento Saludable; Chile: Red Chilena de Programas para el Adulto Mayor; Colombia: Red Colombiana de Envejecimiento Activo y Digno; Ecuador: Red Ecuatoriana de Instituciones Gerontológicas; Honduras: Red Nacional Hondureña de Adultos Mayores; Nicaragua: Fundación Universidad Nicaragüense de la Tercera Edad; Perú: Consorcio Envejecimiento y Desarrollo, Mesa de Trabajo de ONG y Afines Sobre Personas Adultas Mayores, Red Horizontes Villa; República Dominicana: Red por una Vejez Digna; Uruguay: Centro Interinstitucional de Colaboración con el Adulto Mayor.
}

que cumple una función estratégica como vehículo de comunicación entre la población argentina de mayor edad. La Fundación ISALUD es una organización no gubernamental creada en 1991 y acreditada como tal por las Naciones Unidas y por el Centro Nacional de Organizaciones Comunitarias de la Argentina (CENOC). La Fundación impulsa estos esfuerzos en la Argentina a través del Instituto Universitario ISALUD, que ofrece un postgrado en gerontología y desarrolla proyectos de gestión y de servicios a la comunidad.

Chile. La Red Chilena de Programas para el Adulto Mayor (RCPAM), creada en 1994, está constituida por 14 entidades (dos universitarias, tres religiosas, dos de adultos mayores, un centro de formación profesional y seis ONG) que operan en las 13 regiones del país. Desde que se creó la Red Tiempos, la RCPAM opera ad hoc como su secretaría permanente, pero se espera que alguna de las redes afiliadas de otro país tome esa responsabilidad en 2005. En el proyecto también participó el Servicio Nacional del Adulto Mayor (SENAMA) —antes Comité Nacional del Adulto Mayor- del Ministerio Secretaría de la Presidencia, que desde 1995 coordina con éxito los sectores y actores sociales vinculados con los adultos mayores. El SENAMA ofreció capacitación a dirigentes de organizaciones de adultos mayores y funcionarios de los gobiernos regionales en Chile.

Perú. La Mesa de Trabajo de ONG y Afines sobre Personas Adultas Mayores (MTOAPAM) cuenta con nueve redes y 11 entidades afiliadas en Perú, tanto de agrupaciones de adultos mayores como de organizaciones dedicadas a su servicio. Trabajó activamente con el gobierno en la elaboración de los Lineamientos de Política para Personas Adultas Mayores, promulgados en el año 2000. En Perú también funciona el Consorcio Envejecimiento y Desarrollo, que agrupa cuatro ONG dedicadas a los adultos mayores en ese país.

Uruguay. Desde 1991, el Centro Interinstitucional de Colaboración con el Adulto Mayor (CICAM), con el apoyo del Banco de Prevención Social y de otras organizaciones nacionales, realiza cursos y actividades de diferente índole y tiene un servicio de "teléfono solidario" que da apoyo a personas mayores que se sienten solas. Cuenta con 420 socios y 75 voluntarios y está vinculado a la Red Nacional de Adultos Mayores del Uruguay (REDNAMU). En el proyecto participa también el Banco de Previsión Social, que completó un ciclo de capacitación para dirigentes y voluntarios de unas 400 organizaciones que trabajan con esa entidad estatal en la prestación de servicios a la población uruguaya de mayor edad. 


\section{ACTIVIDADES Y PRODUCTOS ESPERADOS}

El proyecto Red Tiempos ha financiado actividades de asistencia técnica y de capacitación en los países participantes y se prevé que, como resultado de este esfuerzo, al finalizar el proyecto en abril de 2005 se hayan alcanzado los siguientes resultados:

\section{A nivel regional y subregional}

1. Plataformas fortalecidas y autosostenibles de servicios (generación y diseminación de información sobre buenas prácticas, asistencia técnica y capacitación) que ayuden a las redes nacionales afiliadas. Las redes nacionales, por su parte, deben crear plataformas y servicios similares para apoyar a las organizaciones nacionales y locales que las integran y fortalecer su capacidad de:

- captar nuevos miembros

- mantener su gestión estratégica y liderazgo

- elaborar y gestionar nuevos proyectos

- coordinar el trabajo con organismos públicos

- recaudar fondos

- promover estilos de vida y políticas para el envejecimiento digno, activo, productivo y saludable en la sociedad

- formar gestores para el desarrollo de microempresas donde trabajen adultos mayores

- crear servicios informativos en el sitio de la Red Tiempos en Internet

2. Más de 3000 organizaciones para adultos mayores de bajos ingresos (en Argentina, 450; en Chile, 2 000; en Perú, 200; en Uruguay, 500) con la capacidad de desarrollar, ofertar y gestionar proyectos y servicios para adultos mayores, así como de recaudar fondos y de mejorar el acceso de los adultos mayores miembros de esas organizaciones a los servicios esenciales

3. Mayor capacidad de coordinación entre las organizaciones participantes y los órganos del Estado

4. Entidades coordinadoras estatales fortalecidas por lo menos en dos países participantes, mientras un tercero debe contar con una propuesta para mejorar la coordinación

5. Cuatro proyectos dirigidos a mejorar la situación de los adultos mayores pobres (tres perfiles de proyecto y una propuesta de préstamo) que pudieran ser presentados al BID.

\section{En los países participantes}

Como resultado de las consultorías realizadas y de la capacitación de personal, las redes nacionales vinculadas a los adultos mayores en los países que participan en el proyecto deben tener mayores posibilidades de coordinación con los organismos del Estado. El trabajo debe estar dirigido no solo a mejorar su capacidad de liderazgo en la sociedad, sino también a desarrollar sus posibilidades de encargarse de organizaciones sociales, planificar y administrar proyectos y servicios, elaborar y diseminar información, mantener programas de enseñanza permanente y de capacitación laboral, apoyar microempresas, trabajar como voluntarios, mejorar la alimentación, las condiciones de vivienda y la atención domiciliaria de los adultos mayores, impulsar la teleasistencia - por ejemplo, la ayuda a domicilio por vía telefónica o por Internet, o el empleo de aparatos especializados como los utilizados para medir la presión arterial a distancia y los desfibriladores por control remoto, entre otros-, programar actividades socioculturales y recreativas de buena calidad y llevar a cabo actividades de asesoría legal, entre otros aspectos. Además, las redes y organizaciones nacionales de apoyo a los adultos mayores deben estar en condiciones de elaborar nuevas propuestas de proyectos que permitan dar continuidad al trabajo desarrollado. Los resultados esperados en los diferentes países participantes son:

En Argentina:

- Contar con una nueva red nacional de redes y organizaciones de adultos mayores de bajos ingresos (Red Mayor), 40 organizaciones fortalecidas y 10 nuevas organizaciones habilitadas para ofrecer servicios en las áreas descritas

- Contar con seis consejos provinciales de adultos mayores, como espacios de reflexión e intercambio de experiencias y de concertación de intereses con el Gobierno para el diseño, la planificación y la ejecución de políticas, estrategias y acciones dirigidas a apoyar a los adultos mayores y a sensibilizar a la comunidad acerca de sus posibilidades

- Presentar dos estudios y perfiles de entidades privadas al BID

En Chile:

- Contar con 2000 dirigentes adultos mayores capacitados por el SENAMA y la RCPAM, incrementar la afiliación mediante una campaña de captación de nuevos miembros

- Tener siete comités regionales de adultos mayores fortalecidos y 350 funcionarios de gobiernos regionales capacitados en materia de gestión descentralizada, captación de fondos para el financiamiento de las iniciativas, mecanismos de coordinación multisectorial y diseño de políticas en apoyo a los adultos mayores

- Presentar al BID una propuesta de préstamo 
En Perú:

- Fortalecer la Mesa de Trabajo de ONG y Afines sobre Personas Adultas Mayores (MTOAPAM) como red nacional, contar con 200 dirigentes de organizaciones de adultos mayores capacitados en gestión organizacional, estrategias implantadas de mercadeo social y captación de nuevos miembros

- Lograr el consenso sobre un plan nacional de acción para personas mayores y difundirlo mediante el Ministerio de Promoción de la Mujer y del Desarrollo Humano (PROMUDEH) y la MTOAPAM

- Contar con un estudio y un perfil de proyecto de continuidad

En Uruguay:

- Capacitar a 200 dirigentes de las redes nacionales de adultos mayores, a 600 dirigentes voluntarios y a 380 dirigentes comunitarios de zonas empobrecidas

- Contar con una red nacional de 429 organizaciones activas bajo la tutela del Banco de Previsión Social

- Fortalecer el CICAM y la REDNAMU, contar con dirigentes de 70 organizaciones de adultos mayores capacitados y con un banco de proyectos

- Capacitar a 30 técnicos del Banco de Previsión Social en la promoción del voluntariado, la gestión de servicios sociales y la formulación de proyectos; contar con voluntarios capacitados y con un estudio que identifique las alternativas de coordinación entre las organizaciones públicas y la sociedad civil de adultos mayores

- Preparar un estudio y un perfil de proyecto de continuidad

\section{RESULTADOS Y EVALUACIÓN}

El proceso evaluativo final de los resultados del proyecto Red Tiempos, programado para abril de 2005, verifica los avances logrados en cada una de las metas propuestas. Además, debe analizar su impacto en las políticas y prácticas de las entidades públicas y privadas vinculadas con la población de adultos mayores en los países participantes y en la Red Tiempos en su conjunto. En este proceso, la evaluación se concentrará en los cambios esperados en cuanto a la proporción de ciudadanos de bajos ingresos que forman parte de los grupos beneficiarios del proyecto, la capacidad estructural y la sostenibilidad de las redes y organizaciones participantes. También debe evaluar el grado de satisfacción de los beneficiarios finales (miembros y usuarios) con los resultados alcanzados por las mismas. Se anticipa que el ciclo de evaluación será parte de un plan estratégico para me- jorar la Red Tiempos, en el que deberán participar todas las redes que forman parte de ella.

De las experiencias desarrolladas hasta la fecha se puede afirmar que los participantes en el proyecto han percibido como productos de valor:

- la internalización de una visión "empresarial" que está cambiando los estilos y las estrategias para conducir sus redes

- la articulación con grupos que tenían poca comunicación entre sí o que no se conocían

- la vinculación de organizaciones del interior de los países participantes

- la creación de habilidades para elaborar propuestas de nuevas iniciativas y crear "bancos de proyectos"

- la valoración de la promoción social - como herramienta de trabajo- del liderazgo de las organizaciones de adultos mayores o que trabajan con ese grupo poblacional

- la constitución de nuevas redes y la renovación o sustitución de las redes débiles o inoperantes

Entre las lecciones aprendidas durante la marcha del proyecto se destaca la necesidad de modificar la idea original de tener una secretaría permanente (central) de la Red Tiempos con una plataforma única de servicios, y pasar a una plataforma descentralizada con ofertas diferenciadas generadas por las distintas redes nacionales que se especializarían en temas específicos. Así, se espera que el apoyo desde Argentina se concentre en la gestión de organizaciones y proyectos, desde Chile en la recaudación de fondos, desde el Perú en la gestación y apoyo de microempresas de personas mayores, y desde Uruguay en la promoción social.

En conclusión, los resultados obtenidos hasta el momento demuestran que el camino de empoderar a la sociedad civil de adultos mayores puede llevar a la inserción socioeconómica y ciudadana de los adultos mayores y a aumentar su participación directa en la tarea de propiciar y pactar con el resto de la sociedad el Nuevo Contrato Social Intergeneracional que exige la nueva etapa de desarrollo y configuración demográfica en que vivimos.

\section{SYNOPSIS}

Empowering older adults who are organized to search for a new social contract: experiences of the Inter-American Development Bank and Red Tiempos

The low priority that is given to old age in national policies in Latin America and the Caribbean compels older adults to 
depend more on their own efforts, organization, and leadership role, as well as on support from their families and the community, in order to meet their basic needs. Various organizations of older adults have gained important ground and benefits in Latin America and the Caribbean. However, the duties and rights of older persons have continued to be limited due to the managerial and financing weaknesses of those organizations, the low level of participation in the organizations by older adults, and limited governmental support. The older-adult organizations should become sustainable entities that are capable of taking on a greater leadership role. The Inter-American Development Bank has funded a project presented by Red Tiempos (Times Network), its branches in Argentina, Chile, Peru, and Uruguay, and other public entities in these countries, with the objective of increasing the capacity and influence of that net- work and its branches. Through this project, the Times Network has created a decentralized platform that provides technical assistance and training to the participating country networks, with the aim of increasing their organizational strength, coordination with governments, and fundraising. The results that have been achieved so far show that empowering the civil society of older adults can lead to their socioeconomic and civic integration and to their increased participation in the task of facilitating the new intergenerational social contract that is required by the new stage of development and the demographic pattern that Latin America and the Caribbean are now experiencing.

Key words: aged, health services for the aged, organizations.

\section{REFERENCIAS}

1. Programa de las Naciones Unidas para el Desarrollo, Banco Interamericano de Desarrollo. Índice de desarrollo. Sociedad civil de Argentina. Buenos Aires: PNUD/ BID; 2000.
2. Banco Interamericano de Desarrollo. Plan de operaciones del Proyecto de Atención a Organizaciones de Adultos Mayores. Washington, D.C.: BID; 2002. (ATN/JF7779-RS).
3. Banco Interamericano de Desarrollo. Directorio de organizaciones de adultos mayores. Washington, D.C.: BID; 2002. Hallado en: http://www.iadb.org/regions/ aging/esp/directory.htm. Acceso el 14 de abril de 2005 